\title{
A METHOD OF PAIRED INTEGRAL EQUATIONS IN THE REGION OF LAPLACE TRANSFORMS FOR SOLVING NONSTATIONARY HEAT CONDUCTION PROBLEMS WITH MIXED DISCONTINUOUS BOUNDARY CONDITIONS
}

\author{
N. I. Yurchuk, V. P. Kozlov, and
}

P. A. Mandrik

UDC 517.968, 536.24

On the basis of the method developed, the solutions of four problems of mathematical physics are obtained for an infinite plate (a plane layer of thickness $z=h$ ) with assignment of mixed discontinuous boundary conditions $(B C)$ on one of the surfaces $z=0$ of the plate and unmixed $B C$ on the other surface $z=h$.

In the present work a development and extension of the method of paired integral equations are suggested to solve parabolic partial differential equations with mixed boundary conditions (BC). A circle $r=R$ on the surface $z=0$ is a line of discontinuity of mixed BC. Mixed discontinuous BC in problems of mathematical physics are taken to mean problems different from those of the Dirichlet and Neumann type. In other words, the term "mixed BC" means that within the limits of variation of one variable (in this case, within the limits of variation of the cylindrical coordinate $r$ on the surface $z=0$ of the plate) heterogeneous (mixed) BC of the first and second kind can operate in the corresponding ranges of variation of this variable (see Fig. 1). On the other plate surface $z=h$ the two types of unmixed BC, namely, either of the first or of the second kind, are considered.

Below we show that a solution of the heat-conduction equation for an infinite plate under the action of mixed $\mathrm{BC}$ on one of its surfaces $z=0$ is reduced in a classical form to a solution of the corresponding integral equations in the region of $L$-transforms.

Problem No. 1. It is required to solve the differential equation

$$
\theta_{r r}(r, z, \tau)+r^{-1} \theta_{r}(r, z, \tau)+\theta_{z z}(r, z, \tau)=a^{-1} \theta_{\tau}(r, z, \tau), 0<r<\infty, 0<z<h, \tau>0,
$$

where $r$ and $z$ are cylindrical coordinates; $\tau$ is the time; $a>0$ is the thermal-diffusivity coefficient; $\theta(r, z, \tau)=T(r$, $z, \tau)-T_{0}\left(T_{0}=\right.$ const is the initial temperature of the infinite plate at $\left.\tau=0\right)$.

Mixed BC for Eq. (1) are given at $z=0$ :

$$
\begin{gathered}
\theta(r, 0, \tau)=f(r, \tau), \quad 0 \leq r<R, \quad z=0, \tau>0 ; \\
-\theta_{z}(r, 0, \tau)=0, R<r<\infty, \quad z=0, \tau>0 .
\end{gathered}
$$

For $f(r, \tau)>0$ we assume the existence of the Laplace integral.

A BC of the first kind is prescribed on the surface $z=h$ :

$$
\theta(r, h, \tau)=0,0 \leq r<\infty, z=h, \tau \geq 0 .
$$

The origin of coordinates $r=z=0$ is selected at the center of the circle $r=R$ on the surface $z=0$.

Belarusian State University, Minsk, Belarus. Translated from Inzhenerno-Fizicheskii Zhurnal, Vol. 72, No. 3, pp. 555-571, May-June, 1999. Original article submitted March 20, 1998. 


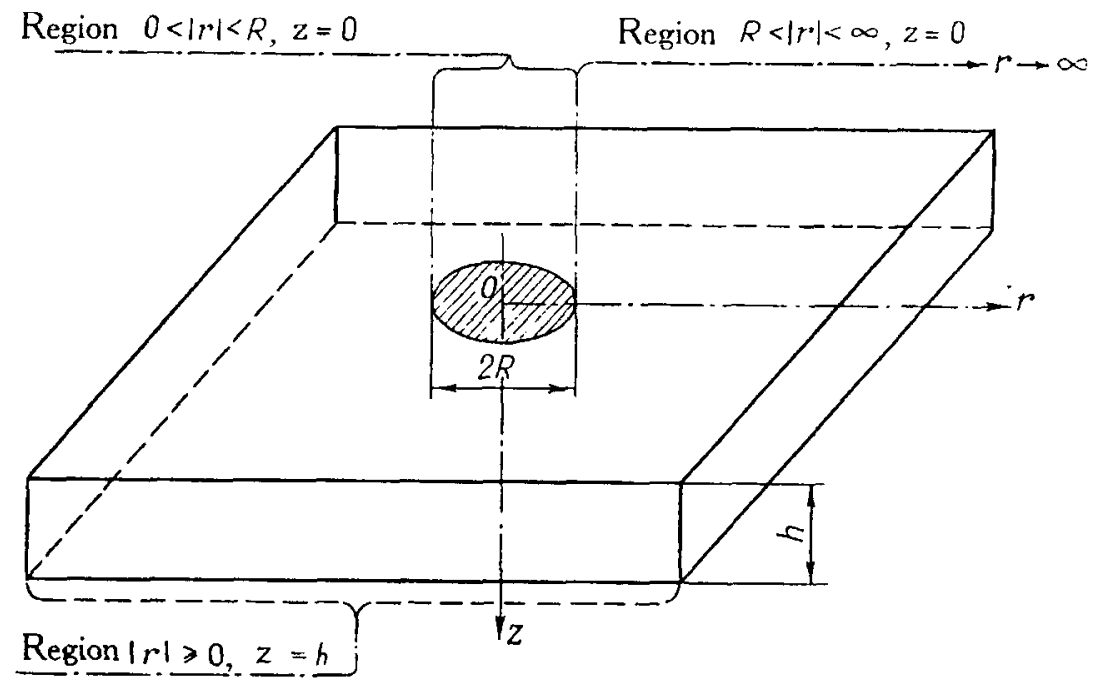

Fig. 1. Diagram notation for regions of boundary conditions in problems Nos. 1-4 on the surfaces of an infinite plate of thickness $h$. In problems Nos. 1 and 3 mixed $B C$ (1.1) and (1.2) are prescribed on the surface $z=0$, and in problems Nos. 2 and 4, mixed BC (2.1) and (2.2) on it. Unmixed BC (1.3) and (3.1) can operate on the other surface $z=h$.

Taking into account boundedness conditions for the temperature on the axis $(r=0,0<z<h, \tau>0)$ and at infinity $(r \rightarrow \infty, 0<z<h, \tau>0)$ and applying the $L$-tranform to Eqs. (1) and (1.1)-(1.3), we present the solution for the transform $\bar{\theta}(r, z, s)=L[\theta(r, z, \tau)]=L\left[\bar{T}(r, z, \tau)-T_{0}\right]$ in the form

$$
\bar{\theta}(r, z, s)=\bar{T}_{0}(r, z, s)-\frac{T_{0}}{s}=\int_{0}^{\infty} \bar{A}(p, s) \frac{\sinh \left[(h-z) \sqrt{\left.\left(p^{2}+\frac{s}{a}\right)\right]}\right.}{\cosh \left[h \sqrt{\left.\left(p^{2}+\frac{s}{a}\right)\right]}\right.} J_{0}(p r) d p, \operatorname{Re} s>0,
$$

where $J_{0}(p r)$ is a Bessel function of the first kind of zero order; $\bar{A}(p, s)$ is the unknown function-transform, which must be determined.

When $h \rightarrow \infty$, from Eq. (1.4) we obtain a solution for $\bar{\theta}(r, z, s)=L\left[\bar{T}(r, z, \tau)-T_{0}\right]$ of a semiinfinite body [1]:

$$
\left.\bar{\theta}(r, z, s)\right|_{h \rightarrow \infty}=\int_{0}^{\infty} \bar{A}(p, s) \exp \left(-z \sqrt{\left.\left(p^{2}+\frac{s}{a}\right)\right) J_{0}(p r) d p .}\right.
$$

and the derivative with respect to the normal to the surface

$$
-\bar{\theta}_{z}(r, z, s)=\int_{0}^{\infty} \bar{A}(p, s) \sqrt{\left(p^{2}+\frac{s}{a}\right)} \frac{\cosh \left[(h-z) \sqrt{\left.\left(p^{2}+\frac{s}{a}\right)\right]}\right.}{\cosh \left[h \sqrt{ }\left(p^{2}+\frac{s}{a}\right)\right]} J_{0}(p r) d p .
$$

at $z=0$ is as follows:

$$
-\bar{\theta}_{z}(r, 0, s)=\int_{0}^{\infty} \bar{A}(p, s) \sqrt{\left(p^{2}+\frac{s}{a}\right) J_{0}(p r) d p}
$$

According to Eq. (1.4), the excess value of the temperature-transform at $z=0$ has the form 


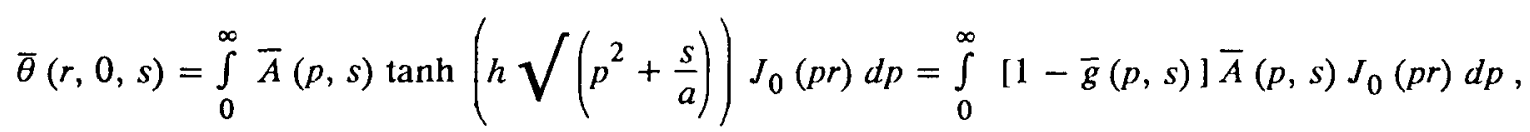

where

$$
\bar{g}(p, s)=\frac{\exp \left(-h \sqrt{ }\left(p^{2}+\frac{s}{a}\right)\right)}{\cosh \left(h \sqrt{ }\left(p^{2}+\frac{s}{a}\right)\right)}
$$

is a known function.

When $h \rightarrow \infty$ and $\bar{g}(p, s) \rightarrow 0$, we find the well-known solution for a half-space at $z=0$ with mixed BC (1.1) and (1.2) [1]. Taking into account mixed $B C$ (1.1) and (1.2) on the surface $z=0$, upon applying the $L$-transform to them, from Eqs. (1.7) and (1.8) we obtain paired integral equations in the region of $L$-transforms, from which the unknown function $\bar{A}(p, s)$ must be determined:

$$
\begin{gathered}
\int_{0}^{\infty}[1-\bar{g}(p, s)] \bar{A}(p, s) J_{0}(p r) d p=\bar{f}(r, s), 0<r<R, \text { Re } s>0 ; \\
\int_{0}^{\infty} \bar{A}(p, s) \sqrt{\left(p^{2}+\frac{s}{a}\right) J_{0}(p r) d p=0,} R<r<\infty, \operatorname{Re} s>0,
\end{gathered}
$$

where the known function is

$$
\bar{f}(r, s)=L[f(r, \tau)]=\int_{0}^{\infty} \exp (-s \tau) f(r, \tau) d \tau, \quad r \in(0, R), \quad z=0, \tau>0
$$

To solve Eqs. (1.10) and (1.11), we introduce another unknown function $\bar{\varphi}_{1}(t, s)$ by means of the relation

$$
\bar{A}(p, s)=\frac{p}{\sqrt{\left(p^{2}+\frac{s}{a}\right)}} \int_{0}^{\infty} \bar{\varphi}_{1}(t, s) \cos \left(t \sqrt{\left.\left(p^{2}+\frac{s}{a}\right)\right)} d t\right.
$$

It turns out that this substitution ensures satisfaction of the homogeneity of one of paired equations (1.11), since substituting Eq. (1.12) into (1.11), we have $(R<r<\infty, \operatorname{Re} s>0)$

$$
\begin{gathered}
\int_{0}^{\infty} \vec{A}(p, s) \sqrt{\left(p^{2}+\frac{s}{a}\right) J_{0}(p r) d p=} \\
=\int_{0}^{\infty} p J_{0}(p r) d p\left\{\bar{\varphi}_{1}(R, s) \frac{\sin \left(R \sqrt{\left.\left(p^{2}+\frac{s}{a}\right)\right)}\right.}{\sqrt{\left(p^{2}+\frac{s}{a}\right)}}-\int_{0}^{R} \frac{\sin \left(t \sqrt{\left.\left(p^{2}+\frac{s}{a}\right)\right)}\right.}{\sqrt{\left(p^{2}+\frac{s}{a}\right)}} \bar{\varphi}_{1 t}^{\prime}(t, s) d t\right\}= \\
=\bar{\varphi}_{1}(R, s) \int_{0}^{\infty} \frac{p J_{0}(p r)}{\sqrt{\left(p^{2}+\frac{s}{a}\right)}} \sin \left(R \sqrt{\left.\left(p^{2}+\frac{s}{a}\right)\right)} d p-\right.
\end{gathered}
$$




$$
-\int_{0}^{R} \bar{\varphi}_{1 t}^{\prime}(t, s) d t \int_{0}^{\infty} \frac{p J_{0}(p r)}{\sqrt{\left(p^{2}+\frac{s}{a}\right)}} \sin \left(t \sqrt{ }\left(p^{2}+\frac{s}{a}\right)\right) d p=0
$$

since $[1-4]$

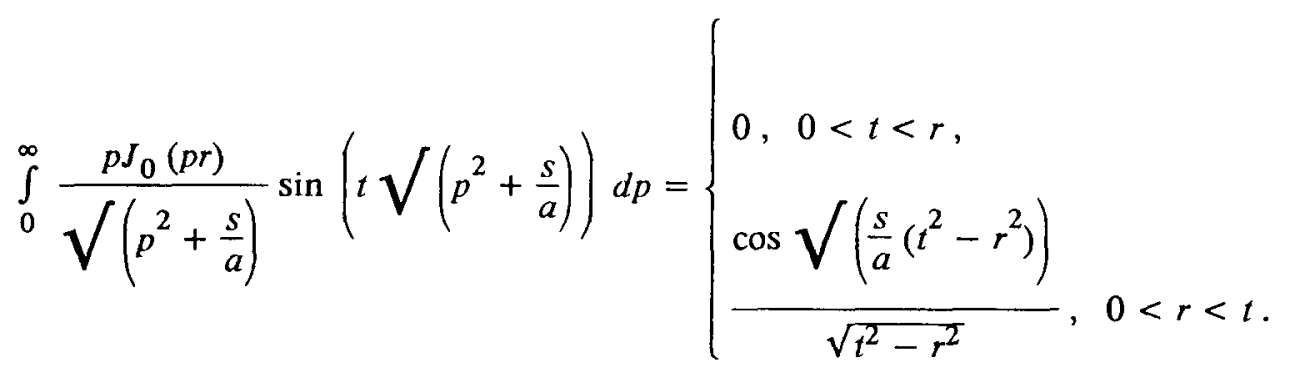

In order to obtain now an equation for determining the function $\bar{\varphi}_{1}(t, s)$, we substitute the value of $\bar{A}(p$, s) given by formula (1.12) into the first paired integral equation (1.10):

$$
\begin{aligned}
& \int_{0}^{\infty} \bar{A}(p, s)[1-\bar{g}(p, s)] J_{0}(p r) d p=\int_{0}^{R} \bar{\varphi}_{1}(t, s) d t \int_{0}^{\infty} \frac{p J_{0}(p r)}{\sqrt{\left(p^{2}+\frac{s}{a}\right)}} \cos \left(t \sqrt{\left.\left(p^{2}+\frac{s}{a}\right)\right)} d p-\right. \\
& -\int_{0}^{R} \bar{\varphi}_{1}(t, s) d t \int_{0}^{\infty} \bar{g}(p, s) \frac{p J_{0}(p r)}{\sqrt{ }\left(p^{2}+\frac{s}{a}\right)} \cos \left(t \sqrt{ }\left(p^{2}+\frac{s}{a}\right)\right) d p= \\
& =\int_{0}^{r} \bar{\varphi}_{1}(t, s) d t \int_{0}^{\infty} \frac{p J_{0}(p r)}{\sqrt{\left(p^{2}+\frac{s}{a}\right)}} \cos \left(t \sqrt{ }\left(p^{2}+\frac{s}{a}\right)\right) d p+ \\
& +\int_{0}^{R} \bar{\varphi}_{1}(t, s) d t \int_{0}^{\infty} \frac{p J_{0}(p r)}{\sqrt{\left(p^{2}+\frac{s}{a}\right)}} \cos \left(t \sqrt{ }\left(p^{2}+\frac{s}{a}\right)\right) d p- \\
& -\int_{0}^{R} \bar{\varphi}_{1}(t, s) d t \int_{0}^{\infty} \bar{g}(p, s) \frac{p J_{0}(p r)}{\sqrt{ }\left(p^{2}+\frac{s}{a}\right)} \cos \left(t \sqrt{ }\left(p^{2}+\frac{s}{a}\right)\right) d p= \\
& =\int_{0}^{r} \bar{\varphi}_{1}(t, s) \frac{\exp \left(\sqrt{\left.\left(\frac{s}{a} \sqrt{r^{2}-t^{2}}\right)\right)}\right.}{\sqrt{r^{2}-t^{2}}} d t-\int_{r}^{R} \bar{\varphi}_{1}(t, s) \frac{\sin \left(\sqrt{\left(\frac{s}{a} \sqrt{t^{2}-r^{2}}\right)}\right)}{\sqrt{\left(t^{2}-r^{2}\right)}} d t- \\
& -\int_{0}^{R} \bar{\varphi}_{1}(t, s) d t \int_{0}^{\infty} \bar{g}(p, s) \frac{p J_{0}(p r)}{\sqrt{ }\left(p^{2}+\frac{s}{a}\right)} \cos \left(t \sqrt{ }\left(p^{2}+\frac{s}{a}\right)\right)=\bar{f}(r, s), 0<r<R, \operatorname{Re} s>0 .
\end{aligned}
$$

In the derivation of Eq. (1.15) the value of the following discontinuous integral $[1-4]$ is used: 


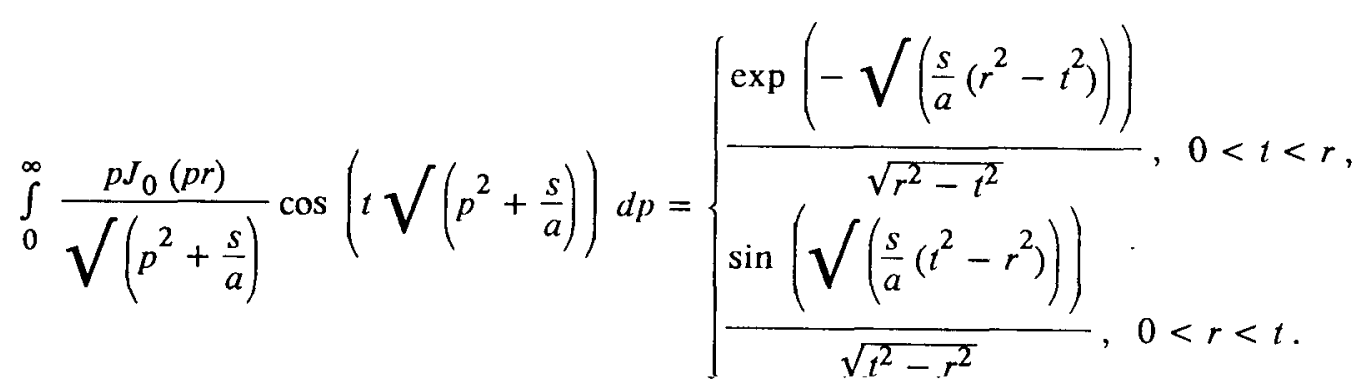

Equation (1.15) is already a basis for determination of the unknown analytic function $\bar{\varphi}_{1}(t, s)$. The solution of this equation can be obtained by representing this function in the form of a functional series [1] expanded in half-integer powers of the parameter $s$. However, in the present work we will find another way to solve this equation.

Let us assume that there is a solution for Eq. (1.15). We reduce this equation to a simpler form, namely, a Fredholm-type equation of the second kind for real variables that contain the complex parameter $s$. For this, we replace $r$ by $\mu$ in formula (1.15), multiply both its sides by the integrating factor cos $\left(\sqrt{s / a\left(r^{2}-\mu^{2}\right)}\right) \cdot 2 \mu d \mu / \sqrt{r^{2}-\mu^{2}}$, and integrate the expression obtained over $\mu$ within the limits from zero to $r$ :

$$
\begin{aligned}
& \int_{0}^{r} \frac{\cos \left(\sqrt{ }\left(\frac{s}{a}\right) \sqrt{r^{2}-\mu^{2}}\right) 2 \mu d \mu}{\sqrt{r^{2}-\mu^{2}}} \int_{0}^{\mu} \frac{\bar{\varphi}_{1}(t, s)}{\sqrt{\mu^{2}-t^{2}}} \exp \left(\sqrt{ }\left(\frac{s}{a}\right) \sqrt{\mu^{2}-t^{2}}\right) d t- \\
& -\int_{0}^{r} \frac{\cos \left(\sqrt{ }\left(\frac{s}{a}\right) \sqrt{r^{2}-\mu^{2}}\right) 2 \mu d \mu}{\sqrt{r^{2}-\mu^{2}}} \times \\
& \times \int_{\mu}^{R} \frac{\bar{\varphi}_{1}(t, s)}{\sqrt{t^{2}-\mu^{2}}} \sin \left(\sqrt{ }\left(\frac{s}{a}\right) \sqrt{t^{2}-\mu^{2}}\right) d t- \\
& -\int_{0}^{r} \frac{\cos \left(\sqrt{\left.\left(\frac{s}{a}\right) \sqrt{r^{2}-\mu^{2}}\right) 2 \mu d \mu}\right.}{\sqrt{r^{2}-\mu^{2}}} \int_{0}^{R} \bar{\varphi}_{1}(t, s) d t \int_{0}^{\infty} \bar{g}(p, s) \times \\
& \times \frac{p J_{0}(p \mu)}{\sqrt{p^{2}+s / a}} \cos \left(t \sqrt{p^{2}+s / a}\right) d p=\int_{0}^{r} \frac{\bar{f}(\mu, s) \cos \left(\sqrt{\left.\left(\frac{s}{a}\right) \sqrt{r^{2}-\mu^{2}}\right) 2 \mu d \mu}\right.}{\sqrt{r^{2}-\mu^{2}}}, 0<r<R, \operatorname{Re} s>0 .
\end{aligned}
$$

In the left-hand side of Eq. (1.17) we change the order of integration by using the well-known Dirichlet formula (for iterated integrals). This leads us to the following integral equation $(0<r<R, \operatorname{Re} s>0$ ):

$$
\begin{aligned}
& \int_{0}^{r} \bar{\varphi}_{1}(t, s) d t-\frac{1}{\pi} \int_{0}^{R} \bar{\varphi}_{1}(t, s) d t\left\{\operatorname{Si}\left[\sqrt{ }\left(\frac{s}{a}\right)(t+r)\right]-\operatorname{Si} \sqrt{ }\left(\frac{s}{a}\right)(t-r)\right]+\int_{0}^{\infty} \bar{g}(p, s) \times \\
& \left.\times \frac{2 \cos \left(t \sqrt{\left.\left(p^{2}+\frac{s}{a}\right)\right)} \sin \left(r \sqrt{ }\left(p^{2}+\frac{s}{a}\right)\right) p d p\right.}{p^{2}+\frac{s}{a}}\right\}=\frac{2}{\pi} \int_{0}^{r} \frac{\bar{f}(\mu, s) \cos \left(\sqrt{ }\left(\frac{s}{a}\right) \sqrt{r^{2}-\mu^{2}}\right) \mu d \mu}{\sqrt{r^{2}-\mu^{2}}},
\end{aligned}
$$


where $\operatorname{Si}(\xi)=\int_{0}^{5}(\sin x / x) d x$ is the integral sine [2-7].

In deriving Eq. (1.18) we use the values of the following integrals:

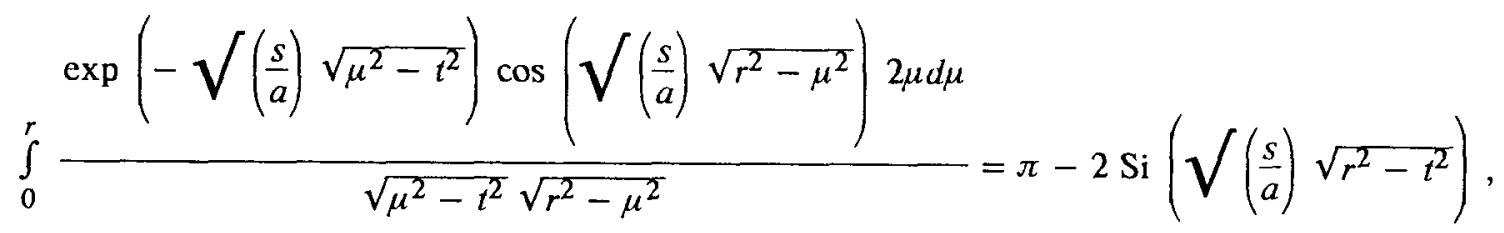

$$
\begin{aligned}
& \int_{0}^{t} \frac{\sin \left(\sqrt{\left.\left(\frac{s}{a}\right) \sqrt{t^{2}-\mu^{2}}\right) \cos \left(\sqrt{ }\left(\frac{s}{a}\right) \sqrt{r^{2}-\mu^{2}}\right) 2 \mu d \mu}\right.}{\sqrt{t^{2}-\mu^{2}} \sqrt{r^{2}-\mu^{2}}}=\operatorname{Si}\left(\sqrt{ }\left(\frac{s}{a}\right)(r+t)\right)+ \\
& +\mathrm{Si}\left(\sqrt{\left(\frac{s}{a}\right)(r-t)}\right)-2 \mathrm{Si}\left(\sqrt{\left.\left(\frac{s}{a}\right) \sqrt{r^{2}-t^{2}}\right)}\right. \\
& \int_{0}^{r} \frac{\sin \left(\sqrt{\left.\left(\frac{s}{a}\right) \sqrt{t^{2}-\mu^{2}}\right) \cos \left(\sqrt{ }\left(\frac{s}{a}\right) \sqrt{r^{2}-\mu^{2}}\right) 2 \mu d \mu}\right.}{\sqrt{t^{2}-\mu^{2}} \sqrt{r^{2}-\mu^{2}}}=\operatorname{Si}\left(\sqrt{ }\left(\frac{s}{a}\right)(t+r)\right)-\operatorname{Si}\left(\sqrt{ }\left(\frac{s}{a}\right)(t-r)\right), \\
& \int_{0}^{r} \frac{\cos \left(\sqrt{\left.\left(\frac{s}{a}\right) \sqrt{r^{2}-\mu^{2}}\right) J_{0}(p \mu) 2 \mu d \mu}\right.}{\sqrt{r^{2}-\mu^{2}}}=\frac{2 \sin \left(r \sqrt{ }\left(p^{2}+\frac{s}{a}\right)\right)}{\sqrt{\left(p^{2}+\frac{s}{a}\right)}} .
\end{aligned}
$$

Differentiation of equality (1.18) with respect to $r$ gives an integral equation in the region of $L$-transforms (with the parameter $s$ ) for determining the unknown function $\bar{\varphi}_{1}(r, s)$ :

$$
\bar{\varphi}_{1}(r, s)-\frac{1}{\pi} \int_{0}^{R} \bar{\varphi}_{1}(\rho, s) \bar{K}_{1}(r, \rho, h, s) d \rho=\bar{F}_{1}(r, s), 0<r<R, \operatorname{Re} s>0
$$

where the kernel of integral equation (1.23) has the form

$$
\begin{aligned}
& \bar{K}_{1}(r, \rho, h, s)=\frac{\sin (\rho-r) \sqrt{s / a}}{\rho-r}+\frac{\sin (\rho+r) \sqrt{s / a}}{\rho+r}+ \\
& +\frac{1}{h} \int_{h \sqrt{s / a}}^{\infty} \frac{\exp (-x)}{\cosh (x)}\left[\cos \left(\frac{\rho-r}{h}\right) x+\cos \left(\frac{\rho+r}{h}\right) x\right] d x
\end{aligned}
$$

and 


$$
\bar{F}_{1}(r, s)=\frac{2}{\pi} \frac{d}{d r} \int_{0}^{r} \frac{\bar{f}(\mu, s) \cos \left(\sqrt{ }\left(\frac{s}{a}\right) \sqrt{r^{2}-\mu^{2}}\right) 2 \mu d \mu}{\sqrt{r^{2}-\mu^{2}}}
$$

is a known function in $(0, R)$ at $z=0$.

Having substituted the value of $\bar{\varphi}_{1}(\rho, s)$ from Eq. (1.23) into (1.12), we find that

$$
\begin{aligned}
& \bar{A}(p, s)=\frac{p}{\sqrt{\left(p^{2}+\frac{s}{a}\right)}} \int_{0}^{R} \cos \left(t \sqrt{ }\left(p^{2}+\frac{s}{a}\right)\right) d t \times \\
& \quad \times\left\{\bar{F}_{1}(t, s)+\frac{1}{\pi} \int_{0}^{R} \bar{\varphi}_{1}(\rho, s) \bar{K}_{1}(t, \rho, h, s) d \rho\right\} .
\end{aligned}
$$

Substitution of Eq. (1.26) into solution (1.4) and application of the inversion formula of the Laplace integral give the value of the temperature field in an infinite plate (i.e., the solution of Eq. (1)) with prescribed mixed $\mathrm{BC}$ (1.1), (1.2) and unmixed BC (1.3) in the form

$$
\begin{gathered}
\theta(r, z, s)=T(r, z, \tau)-T_{0}=\frac{1}{2 \pi i} \int_{\sigma-i \infty}^{\sigma+i \infty} \exp (s \tau) d s \int_{0}^{\infty} \frac{\sinh \left[(h-z) \sqrt{\left.\left(p^{2}+\frac{s}{a}\right)\right]}\right.}{\cosh \left[h \sqrt{\left.\left(p^{2}+\frac{s}{a}\right)\right]} J_{0}(p r) \frac{p d p}{\sqrt{\left(p^{2}+\frac{s}{a}\right)}} \times\right.} \\
\times \int_{0}^{R} \cos \left(t \sqrt{ }\left(p^{2}+\frac{s}{a}\right)\right) d t\left\{\bar{F}_{1}(t, s)+\frac{1}{\pi} \int_{0}^{R} \bar{\varphi}_{1}(\rho, s) \bar{K}_{1}(t, \rho, h, s) d \rho\right\} \\
\tau \geq 0,0 \leq r \leq \infty, 0 \leq z \leq h, \sigma>\operatorname{Re} s>0, \sigma=\text { const, }
\end{gathered}
$$

where $\left.\bar{\varphi}_{1} \varphi, s\right)$ is the solution of integral equation (1.23) with the $L$-parameter $s$.

When $h \rightarrow \infty$, the value of $\bar{\varphi}_{1}(r, s)$ should be sought from integral equation (1.23), the kernel of which will have the form

$$
\lim _{h \rightarrow \infty} \bar{K}_{1}(r, \rho, h, s)=\bar{K}_{1}(r, \rho, s)=\frac{\sin (\rho-r) \sqrt{s / a}}{\rho-r}+\frac{\sin (\rho+r) \sqrt{s / a}}{\rho+r}
$$

and the solution of Eq. (1) for a half-space $(h \rightarrow \infty)$ with assignment of mixed BC (1.1) and (1.2) is written from Eq. (1.27) as follows:

$$
\begin{gathered}
\left.\theta(r, z, s)\right|_{h \rightarrow \infty}=\frac{1}{2 \pi i} \int_{\sigma-i \infty}^{\sigma+i \infty} \exp (s \tau) d s \int_{0}^{\infty} \exp \left(-z \sqrt{\left.\left(p^{2}+\frac{s}{a}\right)\right) \times}\right. \\
\times \frac{p J_{0}(p r) d p}{\sqrt{ }\left(p^{2}+\frac{s}{a}\right)} \int_{0}^{R} \cos \left(t \sqrt{ }\left(p^{2}+\frac{s}{a}\right)\right) d t \times \\
\times\left\{\bar{F}_{1}(t, s)+\frac{1}{\pi} \int_{0}^{R} \bar{\varphi}_{1}(\rho, s) \bar{K}_{1}(t, \rho, s) d \rho\right\}, 0 \leq r \leq \infty, \operatorname{Re} s>0, \tau \geq 0 .
\end{gathered}
$$


When $s \rightarrow 0(\tau \rightarrow \infty)$, from Eq. (1.29) we obtain the well-known stationary solutions of the Laplace equation [8-10] for a half-space with the corresponding mixed BCs at $z=0$.

Problem No. 2. It is required to solve Eq. (1) under unmixed $\mathrm{BC}$ (1.3). The initial temperature field of the infinite plate is uniform and corresponds to problem No. 1, i.e., $T(r, z, 0)=T_{0}=$ const.

The mixed BCs at $z=0$ have the form

$$
\begin{gathered}
-\theta_{z}(r, 0, \tau)=\frac{q(r, \tau)}{\lambda}, 0 \leq r \leq R, \quad z=0, \tau, \lambda>0 \\
\theta(r, 0, \tau)=0, \quad R<r<\infty, \quad z=0, \tau \geq 0
\end{gathered}
$$

where $q(r, \tau)>0$ is a prescribed function of the heat-flux density in the circle $r=R, z=0$ that has the Laplace transform $\bar{q}(r, s)=L[q(r, \tau)] ; \lambda$ is the thermal-conductivity coefficient of the plate.

The solution of Eq. (1) in the region of $L$-transforms with BC (1.3) on the surface $z=h$ is represented by formula (1.4). Taking into account mixed BC (2.1) and (2.2), for determination of the unknown function $\bar{A}(p, s)$ we come to the following paired integral equations:

$$
\begin{gathered}
\int_{0}^{\infty} \bar{A}(p, s) \sqrt{\left(p^{2}+\frac{s}{a}\right) J_{0}(p r) d p=\frac{\bar{q}(r, s)}{\lambda}, 0<r<R, \operatorname{Re} s>0} \\
\int_{0}^{\infty} \bar{A}(p, s) \tanh \left(h \sqrt{\left.\left(p^{2}+\frac{s}{a}\right)\right) J_{0}(p r) d p=0, R<r<\infty,} \operatorname{Re} s>0 .\right.
\end{gathered}
$$

The solution of these equations will be carried out by means of the substitution

$$
A(p, s)=\frac{p}{\sqrt{\left(p^{2}+\frac{s}{a}\right)}} \frac{1}{\tanh \left(h \sqrt{\left(p^{2}+\frac{s}{a}\right)}\right)} \int_{0}^{R} \bar{\varphi}_{2}(t, s) \sin \left(t \sqrt{\left(p^{2}+\frac{s}{a}\right)}\right) d t
$$

Just as for problem No. 1, it is not difficult to show that substitution (2.5) ensures satisfaction of the homogeneity of Eq. (2.4) in accordance with the value of discontinuous integral (1.14).

Substituting Eq. (2.5) into (2.3), we obtain an initial integral equation with the $L$-parameter to find the unknown function $\bar{\varphi}_{2}(t, s)$ under prescribed $\mathrm{BC}(2.1)$ and (2.2) on the plate surface $z=0$ :

$$
\begin{gathered}
\int_{0}^{R} \bar{\varphi}_{2}(t, s) d t\left\{\int _ { 0 } ^ { \infty } \operatorname { s i n } \left(t \sqrt{\left.\left(p^{2}+\frac{s}{a}\right)\right) J_{0}(p r) p d p+}\right.\right. \\
\left.+\int_{0}^{\infty} \frac{\exp \left(-h \sqrt{\left.\left.\left(p^{2}+\frac{s}{a}\right)\right) \sin \left(t \sqrt{ }\left(p^{2}+\frac{s}{a}\right)\right) J_{0}(p r) p d p\right)}\right.}{\sinh \left(h \sqrt{\left.\left(p^{2}+\frac{s}{a}\right)\right)}\right.}\right\}= \\
=\frac{\bar{q}(r, s)}{\lambda}, 0<r<R, \operatorname{Re} s>0 .
\end{gathered}
$$


Just as in problem No. 1, we reduce Eq. (2.6) to an integral equation of the type (1.23). Using the wellknown relation for Bessel functions $p J_{0}(p r)=(1 / r)(d / d r)\left[r J_{1}(p r)\right]$, which we substitute into Eq. (2.6), and integrating its left- and right-hand sides over $r$ within the limits from zero to $r$, we obtain:

$$
\begin{aligned}
& r \int_{0}^{R} \bar{\varphi}_{2}(t, s) d t\left\{\int_{0}^{\infty} \sin \left(t \sqrt{ }\left(p^{2}+\frac{s}{a}\right)\right) J_{1}(p r) d p+\right.
\end{aligned}
$$

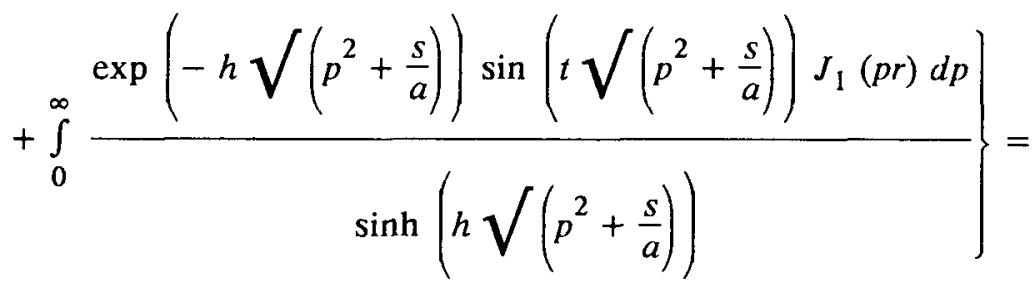

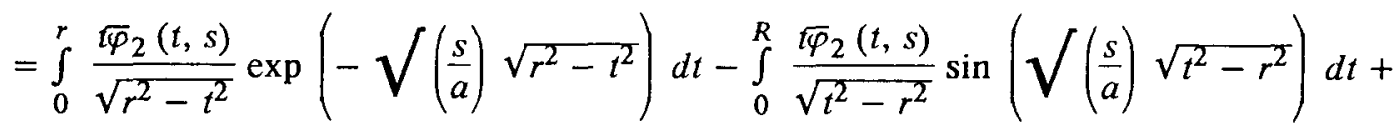

$$
\begin{aligned}
& +\int_{0}^{R} \bar{\varphi}_{2}(t, s) \sin \left(t \sqrt{ }\left(\frac{s}{a}\right)\right) d t+r \int_{0}^{R} \bar{\varphi}_{2}(t, s) d t \times
\end{aligned}
$$

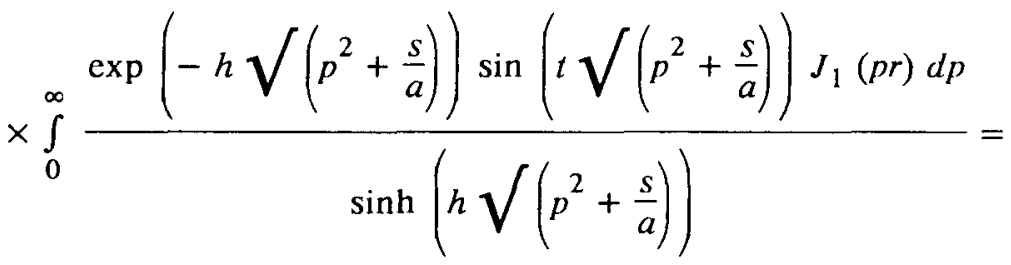

$$
\begin{aligned}
& =\frac{1}{\lambda} \int_{0}^{r} \bar{q}(\rho, s) \rho d \rho, 0<r<R, \text { Re } s>0 .
\end{aligned}
$$

In the derivation of Eq. (2.7) we used the value of the following discontinuous integral [1-4]:

$$
\int_{0}^{\infty} J_{1}(p r) \sin \left(t \sqrt{\left.\left(p^{2}+\frac{s}{a}\right)\right)} d p=\frac{1}{r}\left\{\begin{array}{l}
\sin \left(t \sqrt{\left.\left(\frac{s}{a}\right)\right)+\frac{t \exp \left(-\sqrt{\left(\frac{s}{a}\right)\left(r^{2}-t^{2}\right)}\right)}{\sqrt{r^{2}-t^{2}}}, r>t} ;\right. \\
\sin \left(t \sqrt{\left.\left(\frac{s}{a}\right)\right)}-\frac{t \sin \left(\sqrt{\left.\left(\frac{s}{a}\right)\left(t^{2}-r^{2}\right)\right)}\right.}{\sqrt{t^{2}-r^{2}}, t>r ;}\right.
\end{array}\right.\right.
$$

Integral equation (2.7) can already serve as a basis for determination of the unknown function $\bar{\varphi}_{2}(r, s)$ in the region of $L$-transforms. For this, it is necessary to represent this function as the corresponding functional series expanded in half-integer powers of the parameter $s$, as was done in [1].

Below we suggest another way of determining the unknown function $\bar{\varphi}_{2}(r, s)$. By analogy with problem No. 1, we replace $r$ by $\mu$ in Eq. (2.7), multiply the left- and right-hand sides of Eq. (2.7) by a similar integrating factor to problem No. 1, and then integrate over $d \mu$ within the limits from zero to $r$. As a result, we come to the following integral equation with the $L$-parameter: 


$$
\bar{\varphi}_{2}(r, s)-\frac{1}{\pi} \int_{0}^{R} \bar{\varphi}_{2}(\rho, s) \bar{K}_{2}(r, \rho, h, s) d \rho=\bar{F}_{2}(r, s), 0<r<R, \operatorname{Re} s>0
$$

where

$$
\begin{aligned}
& \bar{K}_{2}(r, \rho, h, s)=\frac{\sin (\rho-r) \sqrt{\left(\frac{s}{a}\right)}}{\rho-r}-\frac{\sin (\rho+r) \sqrt{ }\left(\frac{s}{a}\right)}{\rho+r}- \\
& -\frac{1}{h} \int_{h \sqrt{s / a}}^{\infty} \frac{\exp (-x)}{\sinh (x)}\left[\cos \left(\frac{e-r}{h}\right) x-\cos \left(\frac{\rho+r}{h}\right) x\right] d x
\end{aligned}
$$

the known function $\bar{F}_{2}(r, s) \in(0, R), z=0$ has the form

$$
\bar{F}_{2}(r, s)=\frac{2}{\pi \lambda} \int_{0}^{r} \frac{\bar{q}(\mu, s) \cos \left(\sqrt{\left.\left(\frac{s}{a}\right) \sqrt{r^{2}-\mu^{2}}\right) \mu d \mu}\right.}{\sqrt{r^{2}-\mu^{2}}} .
$$

In deriving Eq. (2.9), we used the values of integrals (1.19)-(1.21) and the calculated iterated integrals

$$
\begin{aligned}
& \frac{1}{\lambda} \int_{0}^{r} \frac{\cos \left(\sqrt{\left.\left(\frac{s}{a}\right) \sqrt{r^{2}-\mu^{2}}\right) 2 \mu d \mu}\right.}{\sqrt{r^{2}-\mu^{2}}} \int_{0}^{\mu} \bar{q}(\rho, s) \rho d \rho= \\
& =\frac{2}{\lambda \sqrt{ }\left(\frac{s}{a}\right)} \int_{0}^{r} \bar{q}(\rho, s) \sin \left(\sqrt{ }\left(\frac{s}{a}\right) \sqrt{r^{2}-\rho^{2}}\right) \rho d \rho, \\
& \int_{0}^{r} \frac{\cos \left(\sqrt{\left.\left(\frac{s}{a}\right) \sqrt{r^{2}-\mu^{2}}\right)}\right.}{\sqrt{r^{2}-\mu^{2}}} 2 \mu^{2} J_{1}(p \mu) d \mu= \\
& =2 p\left\{\frac{\sin \left(r \sqrt{ }\left(p^{2}+\frac{s}{a}\right)\right)-r \sqrt{ }\left(p^{2}+\frac{s}{a}\right) \cos \left(r \sqrt{ }\left(p^{2}+\frac{s}{a}\right)\right)}{\left(p^{2}+\frac{s}{a}\right)^{3 / 2}}\right\} .
\end{aligned}
$$

The value of Eq. (2.11) follows from Eq. (2.12) upon differentiation of the latter with respect to $r$ with allowance for the constant factor $\pi^{-1}$ and cancellation of the left- and right-hand sides of Eq. (2.9) by $r$.

Substitution of the value of $\bar{\varphi}_{2}(t, s)$ from Eq. (2.9) into Eq. (2.5) yields the unknown function $\vec{A}(p, s)$ for the present problem:

$$
\bar{A}(p, s)=\frac{p}{\sqrt{\left(p^{2}+\frac{s}{a}\right)}} \frac{1}{\tanh \left(h \sqrt{\left.\left(p^{2}+\frac{s}{a}\right)\right)}\right.} \int_{0}^{R} \sin \left(t \sqrt{ }\left(p^{2}+\frac{s}{a}\right)\right) d t \times
$$




$$
\times\left\{\bar{F}_{2}(t, s)+\frac{1}{\pi} \int_{0}^{R} \bar{\varphi}_{2}(\rho, s) \bar{K}_{2}(t, \rho, h, s) d \rho\right\} .
$$

Using Eq. (2.14) in solution (1.4) and applying the inversion formula of the Laplace integral, we obtain the value of the temperature field $\theta(r, z, \tau)$ at any point of an infinite plate with assignment of mixed $\mathrm{BC}(2.1)$ and (2.2) at $z=0$ and unmixed $\mathrm{BC}(1.3)$ at $z=h$ :

$$
\begin{gathered}
\theta(r, z, \tau)=T(r, z, \tau)-T_{0}=\frac{1}{2 \pi i} \int_{\sigma-i \infty}^{\sigma+i \infty} \exp (s \tau) d s \times \\
\times \int_{0}^{\infty} \frac{\sinh \left[(h-z) \sqrt{\left.\left(p^{2}+\frac{s}{a}\right)\right]}\right.}{\sinh \left[h \sqrt{ }\left(p^{2}+\frac{s}{a}\right)\right]} \frac{J_{0}(p r) p d p}{\sqrt{\left(p^{2}+\frac{s}{a}\right)}} \times \\
\times \int_{0}^{R} \sin \left(t \sqrt{ }\left(p^{2}+\frac{s}{a}\right)\right) d t\left\{\bar{F}_{2}(t, s)+\frac{1}{\pi} \int_{0}^{R} \bar{\varphi}_{2}(\rho, s) \bar{K}_{2}(t, \rho, h, s) d \rho\right\}, \\
0 \leq r \leq \infty, \tau \geq 0,0 \leq z \leq h, \sigma>\operatorname{Re} s>0, \sigma=\text { const. }
\end{gathered}
$$

where $\bar{\varphi}_{2}(r, s)$ is the solution of integral equation (2.9) with the $L$-parameter $s$.

When $h \rightarrow \infty$, the value of $\bar{\varphi}_{2}(r, s)$ should be determined from integral equation (2.9), the kernel of which will have the form

$$
\lim _{h \rightarrow \infty} \bar{K}_{2}(r, \rho, h, s)=\bar{K}_{2}(r, \rho, s)=\frac{\sin (\rho-r) \sqrt{s / a}}{\rho-r}-\frac{\sin (\rho+r) \sqrt{s / a}}{\rho+r},
$$

and the solution of Eq. (1) for a half-space $(h \rightarrow \infty)$ with assignment of mixed BC (2.1) and (2.2) is written from Eq. (2.15) as

$$
\begin{gathered}
\left.\theta(r, z, \tau)\right|_{h \rightarrow \infty}=\frac{1}{2 \pi i} \int_{\sigma-i \infty}^{\sigma+i \infty} \exp (s \tau) d s \int_{0}^{\infty} \exp \left(-z \sqrt{\left.\left(p^{2}+\frac{s}{a}\right)\right) \times}\right. \\
\times \frac{J_{0}(p r) p d p}{\sqrt{\left(p^{2}+\frac{s}{a}\right)}} \int_{0}^{R} \sin \left(t \sqrt{ }\left(p^{2}+\frac{s}{a}\right)\right) d t \times \\
\times\left\{\bar{F}_{2}(t, s)+\frac{1}{\pi} \int_{0}^{R} \bar{\varphi}_{2}(\rho, s) \bar{K}_{2}(t, \rho, s) d \rho\right\}, 0 \leq r \leq \infty, z \geq 0, \operatorname{Re} s>0, \tau \geq 0 .
\end{gathered}
$$

When $s \rightarrow \infty(\tau \rightarrow \infty)$, from Eq. (2.17) we obtain the well-known stationary solutions of the Laplace equation [8-10] for a half-space with the corresponding mixed BCs at $z=0$.

Problem No. 3. It is required to solve Eq. (1) with mixed BC (1.1) and (1.2) on the surface $z=0$ of a plate. The initial condition is retained as in problems Nos. 1 and 2 . The difference from them will be in the assignment of unmixed $\mathrm{BC}(1.3)$ at $z=h$, which is represented as

$$
\theta_{z}(r, h, \tau)=0,0 \leq r<\infty, \quad z=h, \tau \geq 0,
$$

i.e., when $z=h, 0 \leq r<\infty$, and $\tau \geq 0$, there is ideal heat insulation on this surface of the plate over the entire duration of heat exchange. 
The solution of Eq. (1) in the region of $L$-transforms with mixed BC (1.1) and (1.2) on the plate surface $z=0$ and unmixed $\mathrm{BC}(3.1)$ on the plate surface $z=h$ for the excess temperature-transform $\bar{\theta}(r, z, s)=L[\theta(r, z$, $\tau)$ ] can easily be obtained in the following form:

$$
\bar{\theta}(r, z, s)=\bar{T}(r, z, s)-\frac{T_{0}}{s}=\int_{0}^{\infty} \bar{B}(p, s) \frac{\left[\cosh (h-z) \sqrt{\left(p^{2}+\frac{s}{a}\right)}\right]}{\cosh \left[h \sqrt{ }\left(p^{2}+\frac{s}{a}\right)\right]} J_{0}(p r) d p, \operatorname{Re} s>0
$$

where $\bar{B}(p, s)$ is the unknown function-transform that must be determined.

When $h \rightarrow \infty$, from Eq. (3.2) we obtain solution (1.5) for a half-space [1]. Accounting for mixed BC (1.1) and (1.2) on the plate surface, upon applying the $L$-transformation to them, we come to the following paired integral equations with the $L$-parameter $s$ :

$$
\begin{gathered}
\int_{0}^{\infty} \bar{B}(p, s) J_{0}(p r) d p=\bar{f}(r, s), 0<r<R, \operatorname{Re} s>0 ; \\
\int_{0}^{\infty} \bar{B}(p, s) \sqrt{\left(p^{2}+\frac{s}{a}\right) \tanh \left(h \sqrt{\left.\left(p^{2}+\frac{s}{a}\right)\right)} J_{0}(p r) d p=0, R<r<\infty, \operatorname{Re} s>0,\right.}
\end{gathered}
$$

where the known function-transform is

$$
\bar{f}(r, s)=L[f(r, \tau)], \quad r \in(0, R), \quad z=0, \tau>0
$$

To solve paired equations (3.3) and (3.4) we use the substitution

$$
\bar{B}(p, s)=\frac{p}{\sqrt{\left(p^{2}+\frac{s}{a}\right)}} \frac{1}{\tanh \left(h \sqrt{\left.\left(p^{2}+\frac{s}{a}\right)\right)}\right.} \int_{0}^{R} \bar{\varphi}_{3}(t, s) \cos \left(t \sqrt{\left.\left(p^{2}+\frac{s}{a}\right)\right)} d t,\right.
$$

since it ensures satisfaction of the homogeneity of Eq. (3.4) for $R<r<\infty$.

After the substitution of Eq. (3.5) into Eq. (3.3), we have an integral equation in the region of $L$-transforms for finding the new unknown function $\bar{\varphi}_{3}(r, s)$ :

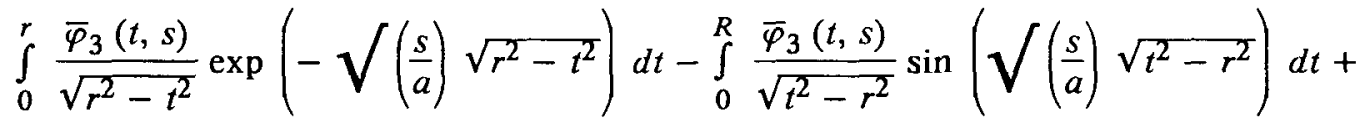

$$
\begin{aligned}
& +\int_{0}^{R} \bar{\varphi}_{3}(t, s) d t \int_{0}^{\infty} \frac{\exp \left(-h \sqrt{\left.\left(p^{2}+\frac{s}{a}\right)\right) \cos \left(t \sqrt{\left(p^{2}+\frac{s}{a}\right)}\right)}\right.}{\sinh \left(h \sqrt{\left.\left(p^{2}+\frac{s}{a}\right)\right)} \sqrt{\left(p^{2}+\frac{s}{a}\right)}\right.} J_{0}(p r) p d p= \\
& =\bar{f}(r, s), \quad 0<r<R, \quad \operatorname{Re} s>0 \text {. }
\end{aligned}
$$

Equation (3.6) is obtained by means of discontinuous integrals (1.14) and (1.16). Using the mathematical method that was applied by us to problem No. 1 , we reduce Eq. (3.6) to an integral equation with the $L$-parameter:

$$
\bar{\varphi}_{3}(r, s)-\frac{1}{\pi} \int_{0}^{R} \bar{\varphi}_{3}(\rho, s) \bar{K}_{3}(r, \rho, h, s) d \rho=\bar{F}_{1}(r, s), 0<r<R, \text { Re } s>0
$$


where the kernel of (3.7) has the form

$$
\begin{gathered}
\bar{K}_{3}(r, \rho, h, s)=\frac{\sin (\rho-r) \sqrt{s / a}}{\rho-r}+\frac{\sin (\rho+r) \sqrt{s / a}}{\rho+r}- \\
-\frac{1}{h} \int_{h \sqrt{s / a}}^{\infty} \frac{\exp (-x)}{\sinh (x)}\left[\cos \left(\frac{\rho-r}{h}\right) x+\cos \left(\frac{\rho+r}{h}\right) x\right] d x
\end{gathered}
$$

The known function $\bar{F}_{1}(r, s) \in(0, R), z=0$ is determined by formula (1.25).

The substitution of the value of $\bar{\varphi}_{3}(t, s)$ from Eq. (3.7) into Eq. (3.5) gives

$$
\begin{gathered}
\bar{B}(p, s)=\frac{p}{\sqrt{\left(p^{2}+\frac{s}{a}\right)}} \frac{1}{\tanh \left(h \sqrt{\left.\left(p^{2}+\frac{s}{a}\right)\right)}\right.} \int_{0}^{R} \cos \left(t \sqrt{\left.\left(p^{2}+\frac{s}{a}\right)\right)} d t \times\right. \\
\quad \times\left\{\bar{F}_{1}(t, s)+\frac{1}{\pi} \int_{0}^{R} \bar{\varphi}_{3}(\rho, s) \bar{K}_{3}(t, \rho, h, s) d \rho\right\} .
\end{gathered}
$$

Using Eq. (3.9) in solution (3.2) and applying the inversion formula of the Laplace integral, we obtain the value of the temperature field $\theta(r, z, \tau)$ at any point of an infinite plate with implementation of mixed $\mathrm{BC}(1.1)$ and (1.2) on the surface $z=0$ of the plate and unmixed $\mathrm{BC}$ of the type (3.1) on the other surface $z=h$ :

$$
\begin{gathered}
\theta(r, z, \tau)=T(r, z, \tau)-T_{0}=\frac{1}{2 \pi i} \int_{\sigma-i \infty}^{\sigma+i \infty} \exp (s \tau) d s \times \\
\times \int_{0}^{\infty} \frac{\cosh \left[(h-z) \sqrt{\left.\left(p^{2}+\frac{s}{a}\right)\right]}\right.}{\sinh \left[h \sqrt{\left.\left(p^{2}+\frac{s}{a}\right)\right]} \frac{J_{0}(p r) p d p}{\sqrt{\left(p^{2}+\frac{s}{a}\right)}} \times\right.} \\
\times \int_{0}^{R} \cos \left(t \sqrt{\left.\left(p^{2}+\frac{s}{a}\right)\right) d t\left\{\bar{F}_{1}(t, s)+\frac{1}{\pi} \int_{0}^{R} \bar{\varphi}_{3}(\rho, s) \bar{K}_{3}(t, \rho, h, s) d p\right\},}\right. \\
0 \leq r \leq \infty, r>0,0 \leq z \leq h, \sigma>\operatorname{Re} s>0, \sigma=\text { const },
\end{gathered}
$$

where $\bar{\varphi}_{3}(r, s)$ is determined from the solution of integral equation (3.7) with the $L$-parameter. When $h \rightarrow \infty$, the value of $\bar{\varphi}_{3}(r, s) \equiv \bar{\varphi}_{1}(r, s)$ (see problem No. 1), and correspondingly the solution of Eq. (1) for a half-space coincides with formula (1.29).

In the stationary state $(\tau \rightarrow \infty), s \rightarrow 0$, we obtain the well-known stationary solutions [8-10] for a semiinfinite body with the corresponding mixed $\mathrm{BC}$ at $z=0$.

Problem No. 4. It is required to solve Eq. (1) with mixed BC (2.1) and (2.2) on the surface $z=0$ of a plate and unmixed BC of the type (3.1) on the other surface $z=h$ of the plate.

The initial condition coincides with problems Nos. 1-3. The solution of Eq. (1) under BC (3.1) is determined by formula (3.2).

Using for Eq. (3.2) mixed $\mathrm{BC}(2.1)$ and (2.2) at $z=0$, upon applying the $L$-transformation to them, we obtain the following paired integral equations with the $L$-parameter for finding the unknown function-transform $\bar{B}(p, s)$ : 


$$
\begin{gathered}
\left.\int_{0}^{\infty} \bar{B}(p, s) \sqrt{\left(p^{2}+\right.} \frac{s}{a}\right) \tanh \left(h \sqrt{\left.\left(p^{2}+\frac{s}{a}\right)\right)} J_{0}(p r) d p=\frac{\bar{q}(r, s)}{\lambda}, 0<r<R, \operatorname{Re} s>0 ;\right. \\
\int_{0}^{\infty} \bar{B}(p, s) J_{0}(p r) d p=0, R<r<\infty, \operatorname{Re} s>0 .
\end{gathered}
$$

To solve these equations, we carry out the substitution

$$
\bar{B}(p, s) \frac{p}{\sqrt{\left(p^{2}+\frac{s}{a}\right)}} \int_{0}^{R} \bar{\varphi}_{4}(t, s) \sin \left(t \sqrt{\left.\left(p^{2}+\frac{s}{a}\right)\right)} d t\right.
$$

which ensures satisfaction of the homogeneity of Eq. (4.2). When $h \rightarrow \infty, \tanh \left(h \sqrt{p^{2}+s / a}\right) \rightarrow 1$ and we come to the corresponding paired integral equations with the $L$-parameter for investigating the temperature fields in a half-space [1] with prescribed $\mathrm{BC}(2.1)$ and (2.2) at $z=0$. Substitution of $\bar{B}(p, s)$ from Eq. (4.3) into Eq. (4.1), performed as in problem No. 2, gives an integral equation with the $L$-parameter for determination of the unknown function-transform $\bar{\varphi}_{4}(r, s)$ :

$$
\begin{aligned}
& \int_{0}^{r} \frac{\tau \varphi_{4}(t, s)}{\sqrt{r^{2}-t^{2}}} \exp \left(-\sqrt{ }\left(\frac{s}{a}\right) \sqrt{r^{2}-t^{2}}\right) d t-\int_{0}^{R} \frac{\tau \varphi_{4}(t, s)}{\sqrt{t^{2}-r^{2}}} \sin \left(\sqrt{ }\left(\frac{s}{a}\right) \sqrt{t^{2}-r^{2}}\right) d t+ \\
& +\int_{0}^{R} \bar{\varphi}_{4}(t, s) \sin \left(t \sqrt{ }\left(\frac{s}{a}\right)\right) d t-r \int_{0}^{R} \bar{\varphi}_{4}(t, s) d t \times \\
& \times \int_{0}^{\infty} \frac{\exp \left(-h \sqrt{\left.\left(p^{2}+\frac{s}{a}\right)\right) \sin \left(t \sqrt{\left.\left(p^{2}+\frac{s}{a}\right)\right)}\right.}\right.}{\cosh \left(h \sqrt{ }\left(p^{2}+\frac{s}{a}\right)\right)} J_{1}(p r) d p= \\
& =\frac{1}{\lambda} \int_{0}^{r} \bar{q}(\mu, s) \mu d \mu, 0<r<R, \operatorname{Re} s>0 .
\end{aligned}
$$

Expression (4.4) is the basic integral equation with the $L$-parameter for finding the unknown function-transform $\bar{\varphi}_{4}(r, s)$. Using the above-described mathematical methods, this expression can be reduced to a simpler form that is similar to the Fredholm form, but with the $L$-parameter:

$$
\bar{\varphi}_{4}(r, s)-\frac{1}{\pi} \int_{0}^{R} \bar{\varphi}_{4}(\rho, s) \bar{K}_{4}(r, \rho, h, s) d \rho=\bar{F}_{2}(r, s), 0<r<R, \operatorname{Re} s>0
$$

where the known function-transform $\bar{F}_{2}(r, s)$ is determined in $(0, R), z=0, \tau>0$, Re $s>0$ by formula (2.11). The kernel $\bar{K}_{4}(r, \rho, h, s)$ is given by the expression

$$
\begin{aligned}
& \bar{K}_{4}(r, \rho, h, s)=\frac{\sin (\rho-r) \sqrt{s / a}}{\rho-r}-\frac{\sin (\rho+r) \sqrt{s / a}}{\rho+r}+ \\
& +\frac{1}{h} \int_{h \sqrt{s / a}}^{\infty} \frac{\exp (-x)}{\cosh (x)}\left[\cos \left(\frac{\rho-r}{h}\right) x-\cos \left(\frac{\rho+r}{h}\right) x\right] d x,
\end{aligned}
$$


Having determined the value of $\bar{\varphi}_{4}(r, s)$ from Eq. (4.5) and substituting $\bar{\varphi}_{4}(t, s)$ into formula (4.3), we find the unknown function-transform:

$$
\begin{gathered}
\bar{B}(p, s)=\frac{p}{\sqrt{\left(p^{2}+\frac{s}{a}\right)}} \int_{0}^{R} \sin \left(t \sqrt{\left.\left(p^{2}+\frac{s}{a}\right)\right) d t \times}\right. \\
\times\left\{\bar{F}_{2}(t, s)+\frac{1}{\pi} \int_{0}^{R} \bar{\varphi}_{4}(\rho, s) \bar{K}_{4}(t, \rho, h, s) d \rho\right\} .
\end{gathered}
$$

Use of Eq. (4.7) in solution (3.2) and application of the inversion formula of the Laplace integral allow us to determine the sought temperature field at any point $0 \leq r \leq \infty, 0 \leq z \leq h, \tau \geq 0$ of an infinite plate with mixed $\mathrm{BC}(2.1)$ and (2.2) on the plate surface $z=0$ and unmixed $\mathrm{BC}$ (3.1) on the other plate surface $z=h$ :

$$
\begin{gathered}
\theta(r, z, \tau)=T(r, z, \tau)-T_{0}=\frac{1}{2 \pi i} \int_{\sigma-i \infty}^{\sigma+i \infty} \exp (s \tau) d s \times \\
\times \int_{0}^{\infty} \frac{\cosh \left[(h-z) \sqrt{\left.\left(p^{2}+\frac{s}{a}\right)\right]} \frac{J_{0}(p r) p d p}{\cosh \left[h \sqrt{\left.\left(p^{2}+\frac{s}{a}\right)\right]}\right.} \times\right.}{\sqrt{\left(p^{2}+\frac{s}{a}\right)}} \\
\times \int_{0}^{R} \sin \left(t \sqrt{\left.\left(p^{2}+\frac{s}{a}\right)\right)} d t\left\{\bar{F}_{2}(t, s)+\frac{1}{\pi} \int_{0}^{R} \bar{\varphi}_{4}(\rho, s) \bar{K}_{4}(t, \rho, h, s) d \rho\right\},\right. \\
0 \leq r \leq \infty, \tau>0,0 \leq z \leq h, \sigma>\operatorname{Re} s>0, \sigma=\text { const },
\end{gathered}
$$

and the value of the function-transform $\bar{\varphi}_{4}(r, s)$ should be determined from the solution of integral equation (4.5) with the $L$-parameter.

When $h \rightarrow \infty$, from Eq. (4.8) we have solution (2.17) for a half-space under mixed BC (2.1) and (2.2) at $z=0$, for $\bar{\varphi}_{4}(r, s) \equiv \bar{\varphi}_{2}(r, s)$, and $\bar{K}_{4}(r, \rho, h, s)$ for $h \rightarrow \infty$ coincides with the value of kernel $(2.16) \bar{K}_{2}(r, \rho, s)$.

The considered problems of mathematical physics with mixed BC are suitable for various practical applications:

a) in designing optimum cooling systems in radioelectronic equipment;

b) in space technology (under conditions of local heating or cooling);

c) in laser medicine for improving the operating safety of laser installations;

d) for control of the laser technology in the production of LIC;

e) in the development of new methods and devices of nondestructive monitoring for identification all the thermophysical characteristics of materials by temperature measurements in time and space directly on a boundary surface of the body under investigation;

f) for solving inverse problems of mathematical physics on the basis of investigation of the direct problem with the aim of optimum regularization.

In the present work for the first time application of the method of paired nonstationary integral equations is implemented for the indicated class of problems of mathematical physics by reducing them to integral equations with an $L$-parameter. A stationary variant of solving the Laplace equation follows from the presented solutions as a particular case with $s \rightarrow 0(\tau \rightarrow \infty)$ [4, 7-9].

The method suggested can also be used to solve the Helmholtz equation $[9,1]$ under the considered mixed $\mathrm{BC}$ in the case of real $k=\operatorname{Re} \sqrt{s / a}$, or to solve the Klein-Gordon equation for pure imaginary $k=i \omega=\operatorname{Im} \sqrt{s / a}$, since the analytical result of their solution leads to identical integral equations (1.29) and (2.17). 
In conclusion we note that practical application of the presented solutions for the mixed problems of mathematical physics with the mixed $\mathrm{BC}$ considered requires, as the final result, determination of the unknown functions-transforms $\bar{\varphi}_{i}(r, s)$ in the complex plane with the prescribed accuracy from integral equations (1.23), (2.9), (3.7), and (4.5). But up to now the theory of solving the latter has not been developed. However, some ideas to solve these integral equations with an $L$-parameter have appeared.

First, they can be solved by representing the analytical function $\bar{\varphi}_{i}(r, s)$ in the form of the corresponding functional power series expanded in half-integer powers of the parameter $s$ [1, 5-7]. Here the prescribed functionstransforms $\bar{f}(r, s)=L[f(r, \tau)]$ and $\bar{q}(r, s)=L[q(r, \tau)]$ in the circle $r=R$ on the surface $z=0$ should be expanded in the corresponding power series in $s$, the expansion coefficients of which will be known. Equating the left and right terms of these expansions in Eqs. (1.23), (2.9), (3.7), and (4.5), for identical powers of the parameter $s$ we obtain a system (infinite or finite) of corresponding integral equations with degenerate kernels, which can be solved by well-known methods [12].

Second, our preliminary investigations of the nonstationary kernels $\bar{K}_{i}(r, \rho, h, s)$ present in Eqs. (1.23), (2.9), (3.7), and (4.5) have shown that they are bounded and square integrable within the intervals $0<r<R$, $0<\rho<R$ for $\operatorname{Re} s>0$, and consequently, in order to solve integral equations (1.23), (2.9), (3.7), and (4.5), one can apply a method of successive approximations [12] developed for a Fredholm equation of the second kind for real variables.

And finally, due attention should be given to approximate computer methods for solving the obtained integral equations with an $L$-parameter by constructing effective computational algorithms.

\section{REFERENCES}

1. N. A. Abdel'razak, Methods of Solving Two-Dimensional Nonstationary Heat Conduction Problems with Mixed and Unmixed Discontinuous Boundary Conditions, Author's Abstract of Candidate's Dissertation in the Physical-Mathematical Sciences, Minsk (1996).

2. A. P. Prudnikov, Yu. A. Brychkov, and O. I. Marichev, Integrals and Series: Elementary Functions [in Russian ], Moscow (1981).

3. A. P. Prudnikov, Yu. A. Brychkov, and O. I. Marichev, Integrals and Series: Special Functions [in Russian ], Moscow (1983).

4. G. N. Watson, Theory of Bessel Functions, Pt. 1 [Russian translation ], Moscow (1949).

5. G. Bateman and A. Erdélyi, Tables of Integral Transformations, Vol. 1. Fourier-Laplace-Mellin Transforms [Russian translation ], Moscow (1969).

6. A. V. Luikov, Theory of Heat Conduction [in Russian ], Moscow (1967).

7. V. P. Kozlov, Two-Dimensional Axisymmetric Nonstationary Heat-Conduction Problems [in Russian ], Minsk (1986).

8. Ya. S. Uflyand, Method of Paired Equations in Problems of Mathematical Physics [in Russian ], Leningrad (1977).

9. N. A. Virchenko, Paired (Triplet) Integral Equations [in Russian ], Kiev (1989).

10. I. Sneddon, Mixed Boundary-Value Problems in Potential Theory, Amsterdam (1966).

11. Yu. V. Gandel', Reduction of a Class of Paired Integral Equations to a Fredholm Equation of the Second Kind, in: Problems of Mathematical Physics and Functional Analysis, Kiev (1976).

12. P. P. Zabreiko, Integral Equations [in Russian], Moscow (1968). 